\title{
SMG6 is the catalytic endonuclease that cleaves mRNAs containing nonsense codons in metazoan
}

\author{
ERIC HUNTZINGER, ${ }^{1}$ ISAO KASHIMA, ${ }^{1}$ MARIA FAUSER, JÉRÔME SAULIÈRE, and ELISA IZAURRALDE \\ Max Planck Institute for Developmental Biology, D-72076 Tübingen, Germany
}

\begin{abstract}
Messenger RNAs harboring nonsense codons (or premature translation termination codons [PTCs]) are degraded by a conserved quality-control mechanism known as nonsense-mediated mRNA decay (NMD), which prevents the accumulation of truncated and potentially harmful proteins. In Drosophila melanogaster, degradation of PTC-containing messages is initiated by endonucleolytic cleavage in the vicinity of the nonsense codon. The endonuclease responsible for this cleavage has not been identified. Here, we show that SMG6 is the long sought NMD endonuclease. First, cells expressing an SMG6 protein mutated at catalytic residues fail to degrade PTC-containing messages. Moreover, the SMG6-PIN domain can be replaced with the active PIN domain of an unrelated protein, indicating that its sole function is to provide endonuclease activity for NMD. Unexpectedly, we found that the catalytic activity of SMG6 contributes to the degradation of PTC-containing mRNAs in human cells. Thus, SMG6 is a conserved endonuclease that degrades mRNAs terminating translation prematurely in metazoa.
\end{abstract}

Keywords: endonuclease; mRNA decay; mRNA surveillance; NMD; PIN domain; UPF1

\section{INTRODUCTION}

In eukaryotes, premature translation termination induced by the presence of nonsense codons (or premature translation termination codons [PTCs]) leads to the assembly of a so-called "surveillance complex" on the mRNA, which targets the transcript for degradation (Conti and Izaurralde 2005; Lejeune and Maquat 2005; Behm-Ansmant and Izaurralde 2006; Rehwinkel et al. 2006). The surveillance complex consists of a distinct set of nonsense-mediated mRNA decay (NMD) effectors, including the evolutionarily conserved proteins UPF1, UPF2, and UPF3 (Conti and Izaurralde 2005; Lejeune and Maquat 2005; Behm-Ansmant and Izaurralde 2006; Rehwinkel et al. 2006). Additional NMD effectors in metazoa include SMG1, a phosphatidylinositol 3-kinase-like kinase that phosphorylates UPF1, and the SMG5-7 proteins, which trigger its dephosphorylation (Hodgkin et al. 1989; Cali et al. 1999; Page et al. 1999; Denning et al. 2001; Pal et al. 2001; Yamashita et al. 2001; Anders et al. 2003; Chiu et al. 2003; Ohnishi et al. 2003; Fukuhara et al. 2005).

\footnotetext{
${ }^{1}$ These authors contributed equally to this work.

Reprint requests to: Elisa Izaurralde, Max Planck Institute for Developmental Biology, Spemannstrasse 35, D-72076 Tübingen, Germany; e-mail: elisa.izaurralde@tuebingen.mpg.de; fax: +49-7071-601-1353,

Article published online ahead of print. Article and publication date are at http://www.rnajournal.org/cgi/doi/10.1261/rna.1386208.
}

Current models for NMD suggest that UPF1 and SMG1 are recruited by ribosomes terminating translation prematurely through interactions with the eukaryotic release factors eRF1 and eRF3 (Czaplinski et al. 1998; Kashima et al. 2006). In the presence of UPF2 and/or UPF3, presumably bound to the mRNA, SMG1 phosphorylates UPF1, which then recruits SMG5, 6, and 7 (Denning et al. 2001; Pal et al. 2001; Yamashita et al. 2001; Anders et al. 2003; Chiu et al. 2003; Ohnishi et al. 2003; Fukuhara et al. 2005). These three related proteins bind phosphorylated UPF1 through a common 14-3-3-like domain (Fukuhara et al. 2005). SMG5 and SMG7 provide a molecular link between the surveillance complex and the general mRNA decay machinery, thereby coupling UPF1 phosphorylation to mRNA degradation (Unterholzner and Izaurralde 2004). The role of SMG6 remained unclear. This protein is required for NMD (Hodgkin et al. 1989; Page et al. 1999; Gatfield et al. 2003; Paillusson et al. 2005; Luke et al. 2007) and is characterized by a C-terminal PilT N-terminus (PIN) domain, which exhibits nuclease activity on singlestranded RNA in vitro (Glavan et al. 2006).

We have shown previously that the first step in the decay of nonsense-codon-containing mRNAs in Drosophila melanogaster is an endonucleolytic cleavage occurring in the vicinity of the PTC (Gatfield and Izaurralde 2004). The identity of the endonuclease responsible for this cleavage has remained elusive. Here we show that SMG6 is the long 
sought NMD endonuclease. The catalytic activity resides on its C-terminal PIN domain, which is also present in other proteins with nuclease activity. We show further that an SMG6 protein carrying a catalytically active PIN domain derived from an unrelated protein restores NMD in cells depleted of endogenous SMG6. This indicates that the sole function of the SMG6-PIN domain is to provide endonuclease activity. Finally, and contrary to expectation, we show that the catalytic activity of SMG6 is required for the degradation of PTC-containing mRNAs in human cells. Together, these results definitively demonstrate that SMG6 functions as an endonuclease in the NMD pathway.

\section{RESULTS AND DISCUSSION}

\section{A catalytically active SMG6-PIN domain is required for NMD}

We have shown previously that SMG5 and SMG6 share a C-terminal PIN domain, present in proteins with nuclease activity (Glavan et al. 2006). The SMG5-PIN domain lacks key catalytic residues, whereas the SMG6-PIN domain has the canonical triad of acidic residues crucial for catalysis and exhibits nuclease activity on single-stranded RNA in vitro (Glavan et al. 2006). We hypothesized that SMG6 could be responsible for the endonucleolytic cleavage of nonsense mRNAs in D. melanogaster (Glavan et al. 2006).

To test this hypothesis, we designed a complementation assay in which endogenous SMG6 was depleted from D. melanogaster S2 cells using a specific siRNA (SMG6-siRNA). An siRNA complementary to the bacterial $\beta$-galactosidase mRNA served as a negative control $(\beta$ Gal-siRNA). Depleting SMG6 inhibits NMD (Gatfield et al. 2003). We then asked whether we could restore NMD by expressing wild-type SMG6 or a variant in which two aspartic residues required for catalysis were substituted with asparagines (D881N, D918N; SMG6 mut). Transcripts encoding SMG6 proteins were made resistant to the siRNA by introducing mutations disrupting base-pair interactions with this siRNA without altering the protein sequence.

We used three different NMD reporters in this study (Fig. 1A). One reporter is based on a fusion between the open reading frames (ORFs) of Renilla luciferase and green fluorescent protein (R-Luc-GFP). The natural R-Luc stop codon is interpreted as a nonsense codon in this fusion and triggers NMD (R-Luc-GFP-PTC). The reduction in mRNA abundance is reflected by the corresponding decrease in RLuc activity (Fig. 1B).

The additional reporters were described before and derive from the $D$. melanogaster alcohol dehydrogenase (Adh) or the prokaryotic chloramphenicol acetyl transferase (CAT) genes, respectively (Fig. 1A; Gatfield et al. 2003; Gatfield and Izaurralde 2004; Behm-Ansmant et al. 2007). Nonsense codons at positions 64 and 72 of the Adh and CAT ORFs, respectively (Adh-PTC or CAT-PTC), trigger NMD, leading to a strong reduction of mRNA levels relative to the expression of the corresponding wildtype transcripts (Fig. 2; Gatfield et al. 2003; Gatfield and Izaurralde 2004; Behm-Ansmant et al. 2007).

Transfection of SMG6-siRNA into S2 cells suppresses $\mathrm{NMD}$, so the levels of the PTC-containing reporters increased (Figs. 1C,D, 2A,B). When compared to the corresponding wild-type reporters, the up-regulation of
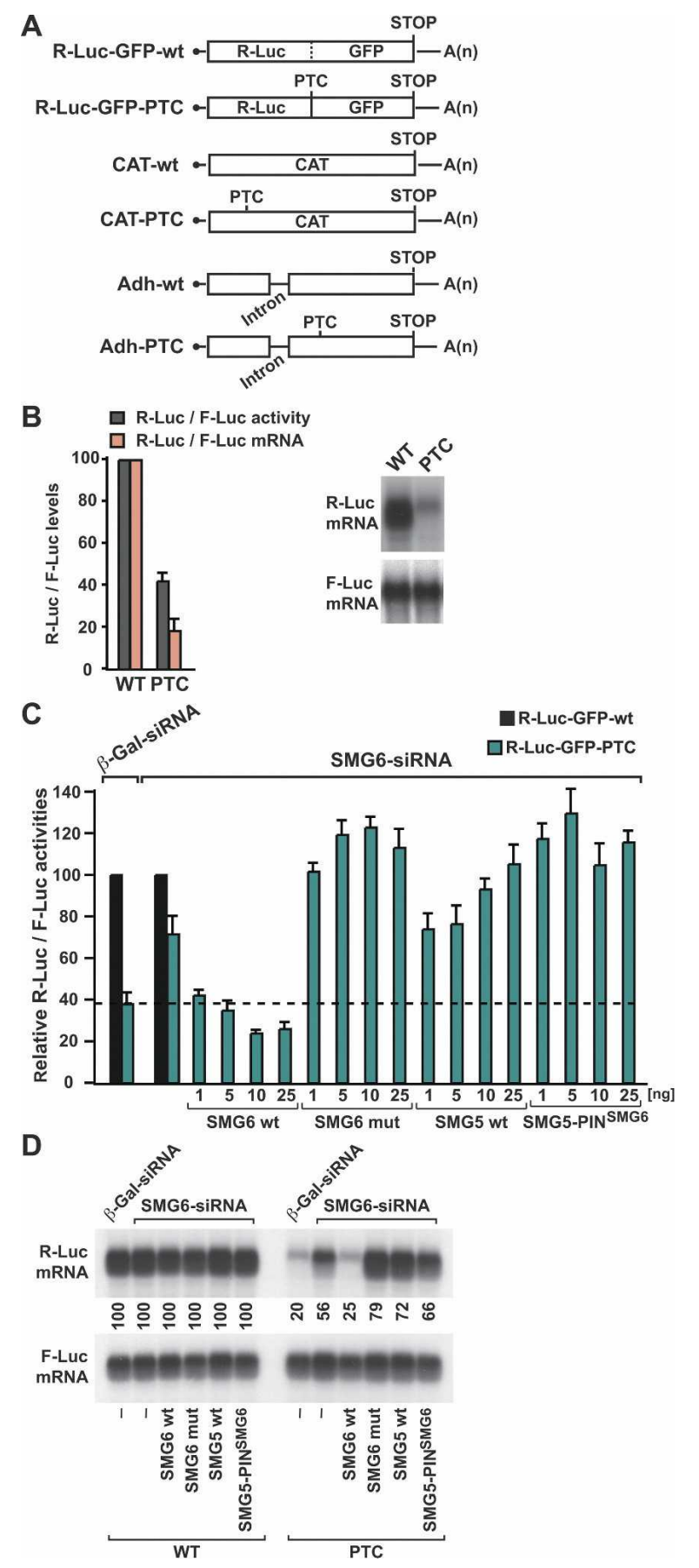

FIGURE 1. (Legend on next page) 
PTC-containing mRNA reporters ranged between 2.5- and sevenfold (Figs. 1C,D, 2A,B). The expression of the siRNAresistant version of SMG6 (SMG6 wt) restored NMD in a concentration-dependent manner, indicating that the effects of the SMG6-siRNA are specific (Figs. 1C,D, $2 \mathrm{~A}, \mathrm{~B})$. In contrast, the SMG6 mutant failed to rescue NMD (Figs. 1C,D, 2A,B; SMG6 mut). Furthermore, this mutant inhibits NMD in a dominant negative manner when overexpressed (Fig. 1C,D; Glavan et al. 2006). The expression levels of wild-type and mutant SMG6 proteins were comparable (Fig. 2C, lanes 2,3). Together these results indicate that a catalytically active SMG6-PIN domain is required for NMD. In agreement with this, an SMG6 protein carrying the PIN domain of SMG5, which lacks two of the three active-site aspartates (Glavan et al. 2006), did not restore NMD (data not shown).

\section{The sole function of the SMG6-PIN domain is to provide endonuclease activity}

If the sole function of the SMG6-PIN domain were to provide endonuclease activity, then any active PIN domain could potentially substitute for that of SMG6. We therefore investigated whether we could replace the SMG6-PIN domain with the PIN domain of NOB1, which has a similar fold and was shown to be catalytically active (Fatica et al. 2003, 2004). An SMG6 protein carrying the NOB1-PIN domain (SMG6-PIN ${ }^{\mathrm{NOB} 1}$ ) rescued NMD in cells depleted of endogenous SMG6 (Fig. 3A-C). Similar results were obtained with the three reporters (Fig. 3A-C). Again in this hybrid protein, substitution of two catalytic residues with asparagines (NOB1 residues D87 and D105, at equivalent positions as D881 and D918 in the SMG6-PIN domain) was sufficient to abolish NMD activity (Fig. 3A-C;

FIGURE 1. Mutations of catalytic residues in the SMG6-PIN domain abolish NMD. (A) Schematic representation of the NMD reporters used in this study. The position of the nonsense codon (PTC) is indicated. (B) S2 cells were transfected with a mixture of two plasmids: one expressing the R-Luc-GFP reporter with or without PTC as indicated; and another expressing Firefly luciferase (F-Luc) as a transfection control. R-Luc activity (gray bars) and RNA levels (orange bars) were normalized to those of the F-Luc control and set to 100 for the wild-type reporter. Mean values and SD from three independent experiments are shown. The right panel shows Northern blot analysis of representative RNA samples. $(C, D)$ S2 cells were transfected with the indicated siRNAs plus the mixture of plasmids described in $B$. Increasing amounts of plasmids encoding siRNAresistant versions of wild-type or mutant SMG6 proteins were included in the transfection mixtures, as indicated. Alternatively, SMG5 wt or a variant carrying the SMG6-PIN domain were also tested. R-Luc activity was normalized to that of the F-Luc and set to 100 for the wild-type reporter (black bars). Mean values and SD from three independent experiments are shown. The dashed line indicates NMD levels in control cells (treated with $\beta$-Gal-siRNA). (D) Northern blot analysis of representative RNA samples. Reporter mRNA levels were normalized to that of the F-Luc mRNA. For each condition, the normalized values of the wild-type reporter were set to 100 .
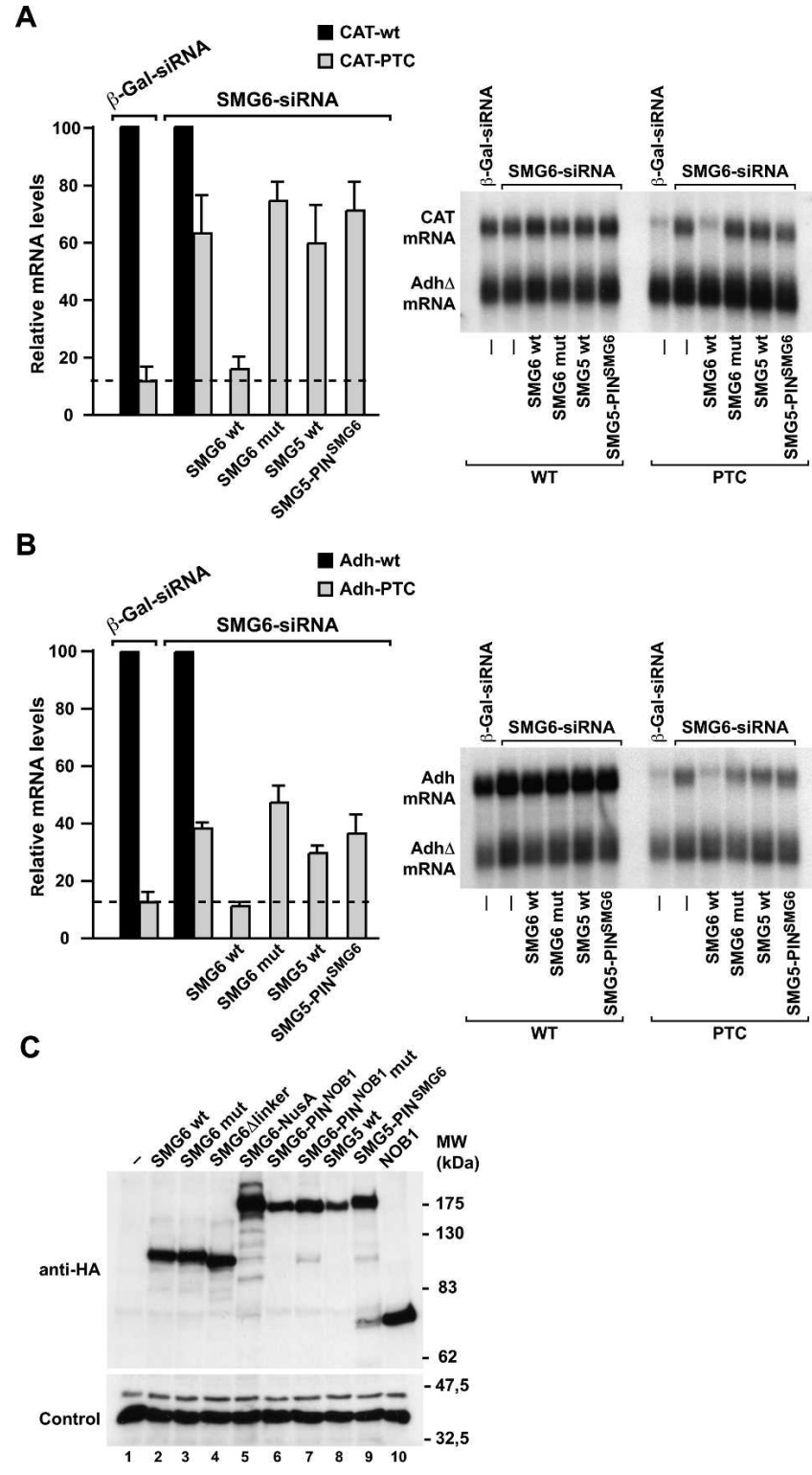

FIGURE 2. A catalytically active SMG6-PIN domain is required for NMD. $(A, B)$ S2 cells were transfected with the indicated siRNAs plus a mixture of two plasmids: one expressing the Adh or CAT reporters with or without PTC as indicated, and another expressing a truncated version of $\operatorname{Adh}(\operatorname{Adh} \Delta)$ as a transfection control. Plasmids encoding SMG5 proteins or siRNA-resistant versions of SMG6 were included in the transfection mixtures, as indicated. Reporter mRNA levels were normalized to that of the Adh $\Delta$ mRNA. For each condition, the normalized values of the wild-type reporter were set to 100 (black bars). Mean values and SD from three independent experiments are shown. The right panels show Northern blot analysis of representative RNA samples. (C) Recombinant SMG5, SMG6, and NOB1 proteins have an HA-tag, so that their expression could be analyzed by Western blotting using an anti-HA antibody. Cotransfected Renilla luciferase (fused C-terminally to a V5 epitope) served as a loading control.

SMG6-PIN $\left.{ }^{\text {NOB1-mut }}\right)$. Similar results were obtained when a single catalytic residue (NOB1 residue D15) was substituted with asparagine (data not shown). Overexpressing NOB1 alone did not affect reporter expression, indicating that the 
A

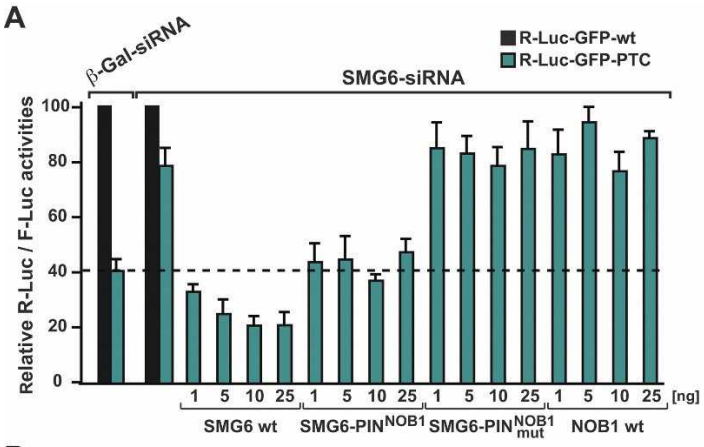

B

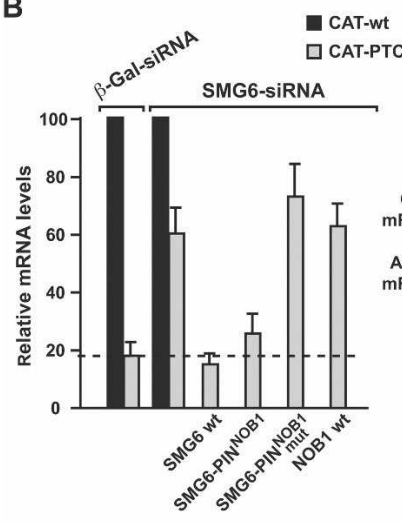

C
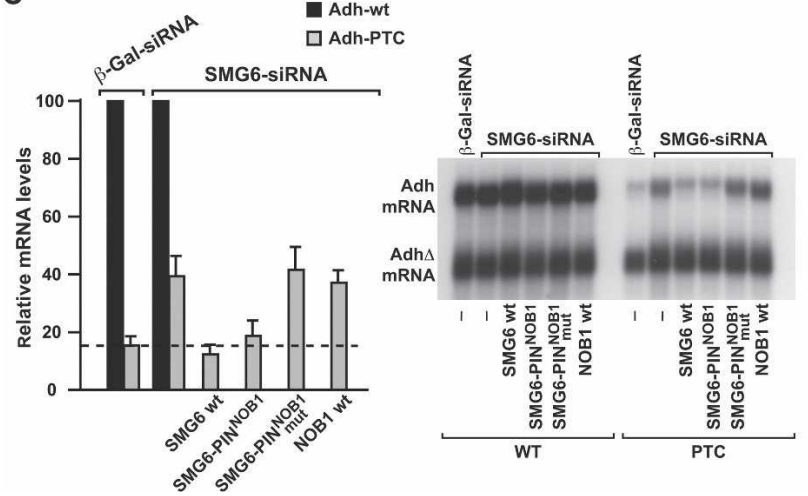

FIGURE 3. The sole function of the SMG6-PIN domain is to provide endonuclease activity. (A) S2 cells were transfected with the indicated siRNAs and a mixture of plasmids as described in Figure 1B. Increasing amounts of plasmids encoding siRNA-resistant versions of wild-type or mutant SMG6 proteins were included in the transfection mixtures, as indicated. Alternatively, full-length NOB1 was tested. R-Luc activity and mRNA levels were analyzed as described in Figure 1C,D. $(B, C)$ S2 cells were transfected with the indicated siRNAs plus the Adh or CAT reporters as described in Figure 2A,B. Plasmids encoding NOB1 or siRNAresistant versions of wild-type or mutant SMG6 proteins were included in the transfection mixtures, as indicated. Reporter mRNA levels were analyzed as described in Figure 2A,B. linker region whose length varies across species $(\sim 52$ residues in $D$. melanogaster; Gatfield et al. 2003; Fukuhara et al. 2005). The 14-3-3-like domain binds phosphorylated UPF1; in doing so it positions the PIN domain in the vicinity of the PTC, so that the mRNA is cleaved at this position. We speculate that the ability of the SMG6-PIN domain to cleave PTC-containing mRNAs may be influenced by the length of the linker region. To investigate the role of this region we generated an SMG6 protein deleted for these sequences (amino acids 676-728). This protein restored NMD in cells depleted of endogenous SMG6 (Fig. 4A-C; SMG6 4 linker), indicating that the linker region is not necessary for NMD.

Next, we replaced the linker region with an unrelated large bacterial protein NusA (498 amino acids). The resulting hybrid protein was active in NMD (Fig. 4A-C; SMG6-NusA). Thus, altering the linker region between the 14-3-3-like and the PIN domains of SMG6 has no detectable effect on NMD efficiency.

\section{The accumulation of decay intermediates correlates with SMG6 catalytic activity}

We have shown before that endonucleolytic cleavage of PTC-containing mRNAs generates $5^{\prime}$ and $3^{\prime}$ decay intermediates, which are rapidly degraded from the newly generated ends by the exosome and XRN1, respectively (Gatfield and Izaurralde 2004). Thus, the $5^{\prime}$ decay intermediate can only be detected in cells in which exosome activity is inhibited (Gatfield and Izaurralde 2004; Behm-Ansmant et al. 2007). This can be achieved by depleting SKI8, a protein required for exosome recruitment and function (Gatfield and Izaurralde 2004;

protein does not degrade RNAs unspecifically (Fig. 3A-C; NOB1 wt). Taken together these results indicate that the role of the SMG6-PIN domain in NMD is to provide endonuclease activity.

\section{The linker region of SMG6 is dispensable for NMD}

The C-terminal PIN domain of SMG6 is connected to a central 14-3-3-like domain through a low-complexity
Houseley et al. 2006; Behm-Ansmant et al. 2007). Conversely, the $3^{\prime}$ decay intermediate can only be detected in cells depleted of XRN1 (Gatfield and Izaurralde 2004; Behm-Ansmant et al. 2007).

By depleting SKI8 or XRN1 we could investigate whether the accumulation of $5^{\prime}$ and $3^{\prime}$ decay intermediates is inhibited in cells lacking a catalytically active form of SMG6. Furthermore, it was of interest to examine whether the position of the cleavage site was altered in cells 
A

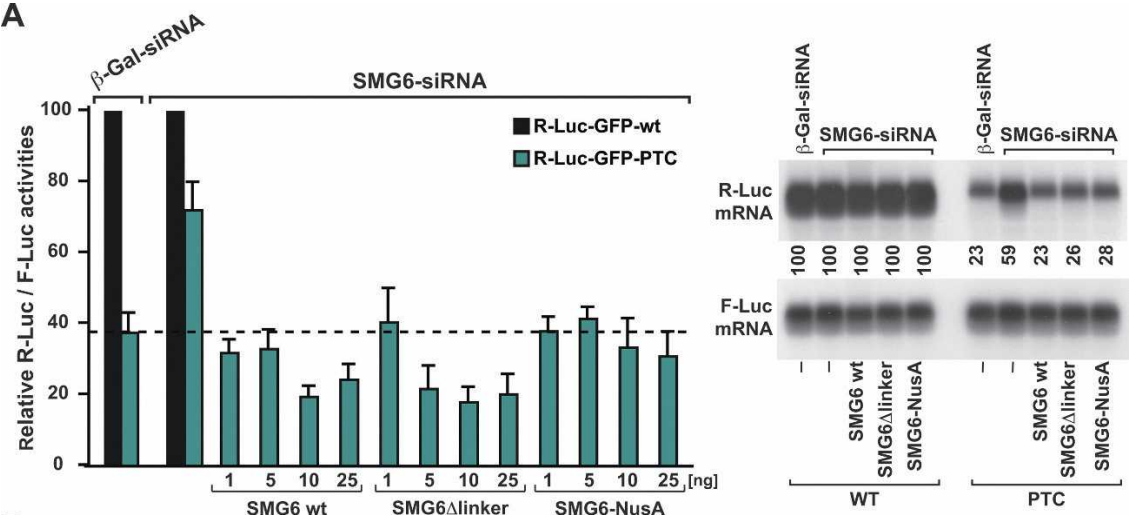

B

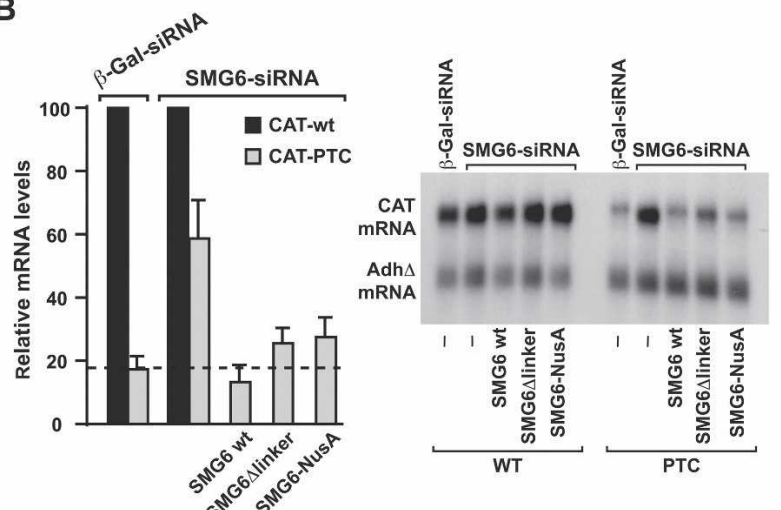

C

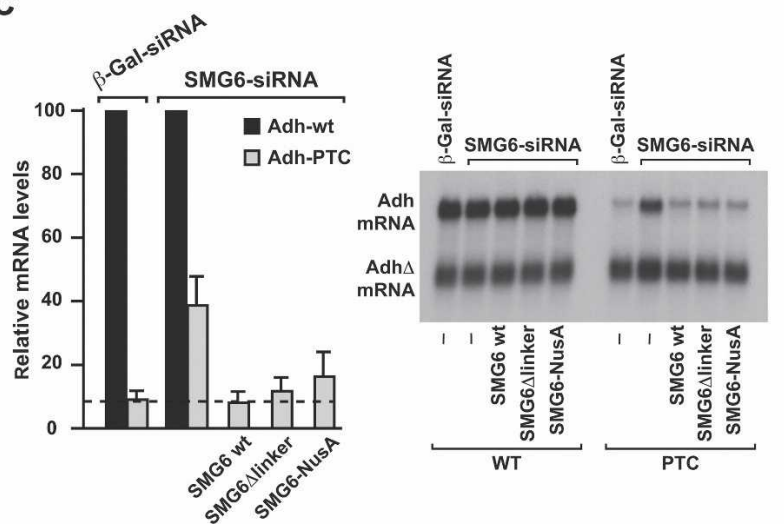

FIGURE 4. The SMG6 linker region is dispensable for NMD. (A) S2 cells were transfected with the indicated siRNAs plus the R-Luc-GFP reporter as described in Figure 1B. Increasing amounts of plasmids encoding siRNA-resistant versions of wild-type or mutant SMG6 proteins were included in the transfection mixtures. R-Luc activity and mRNA levels were analyzed as described in Figure 1C,D. $(B, C)$ S2 cells were transfected with the indicated siRNAs plus the Adh or CAT as described in Figure 2A,B. Plasmids encoding siRNA-resistant versions of wild-type or mutant SMG6 proteins were included in the transfection mixtures, as indicated. Reporter mRNA levels were analyzed as described in Figure 2A,B.

expressing SMG6 proteins in which the distance between the 14-3-3-like and PIN domains was altered by deletion of the linker region or insertion of the NusA protein.

In cells depleted of SKI8 or XRN1, the $5^{\prime}$ or $3^{\prime}$ decay intermediates, respectively, accumulated as shown before (Fig. 5A,B, lanes 22,36, asterisks; Gatfield and Izaurralde 2004; Behm-Ansmant et al. 2007). The decay intermediates were not detected in cells depleted of SKI8 or XRN1, when
NMD was inhibited by depleting endogenous SMG6 and expressing the catalytically inactive SMG6 mutant (Fig. 5A,B, lanes 23,37$)$. In contrast, the full-length PTC-containing transcript accumulated in these cells, showing a precursorproduct relationship between the fulllength mRNA and the decay intermediates (Fig. 5A,B, lanes 23,37). Expressing wild-type SMG6 in cells depleted of both SMG6 and SKI8, or SMG6 and XRN1, restored NMD as described above, with an increase in levels of the decay intermediates and a decrease in those of the full-length PTC reporter (Fig. 5A,B, lanes 24,38).

Similarly, in cells lacking endogenous SMG6 and depleted of either SKI8 or XRN1, the expression of an SMG6 protein carrying the NOB1-PIN domain led to the accumulation of the decay intermediates and the corresponding decrease of the full-length transcript (Fig. 5A,B, lanes 25,39), whereas expressing SMG6 fused to a catalytically inactive NOB1-PIN domain strongly reduced decay intermediate levels and increased those of the full-length transcript (Fig. 5A,B, lanes 26,40).

Expressing SMG6Dlinker or SMG6NusA in cells depleted of SKI8 and SMG6 or XRN1 and SMG6 restored NMD and the accumulation of the decay intermediates. The electrophoretic mobility of the decay intermediates was, however, comparable to that observed in control cells (Fig. 5A,B, cf. lanes 27,28 and lane 22, cf. lanes 41,42 and lane 36), suggesting that the position of the endonucleolytic cleavage is not influenced by the protein sequences connecting the 14-3-3-like and the PIN domains of SMG6. Nonetheless, there must be some constraints on the recruitment and positioning of the SMG6PIN domain during the assembly of the surveillance complex because, when this domain was inserted in the SMG5 protein, it did not rescue NMD in cells depleted of endogenous SMG6 (Figs. 1C,D, 2A,B; SMG5-PIN ${ }^{\text {SMG6}}$ ).

In summary, the accumulation of the decay intermediates depends on the catalytic activity of SMG6 and correlates with the degradation of the full-length transcript (Fig. 5A,B), indicating that the intermediates are generated from the fulllength mRNA undergoing endonucleolytic cleavage by SMG6. 


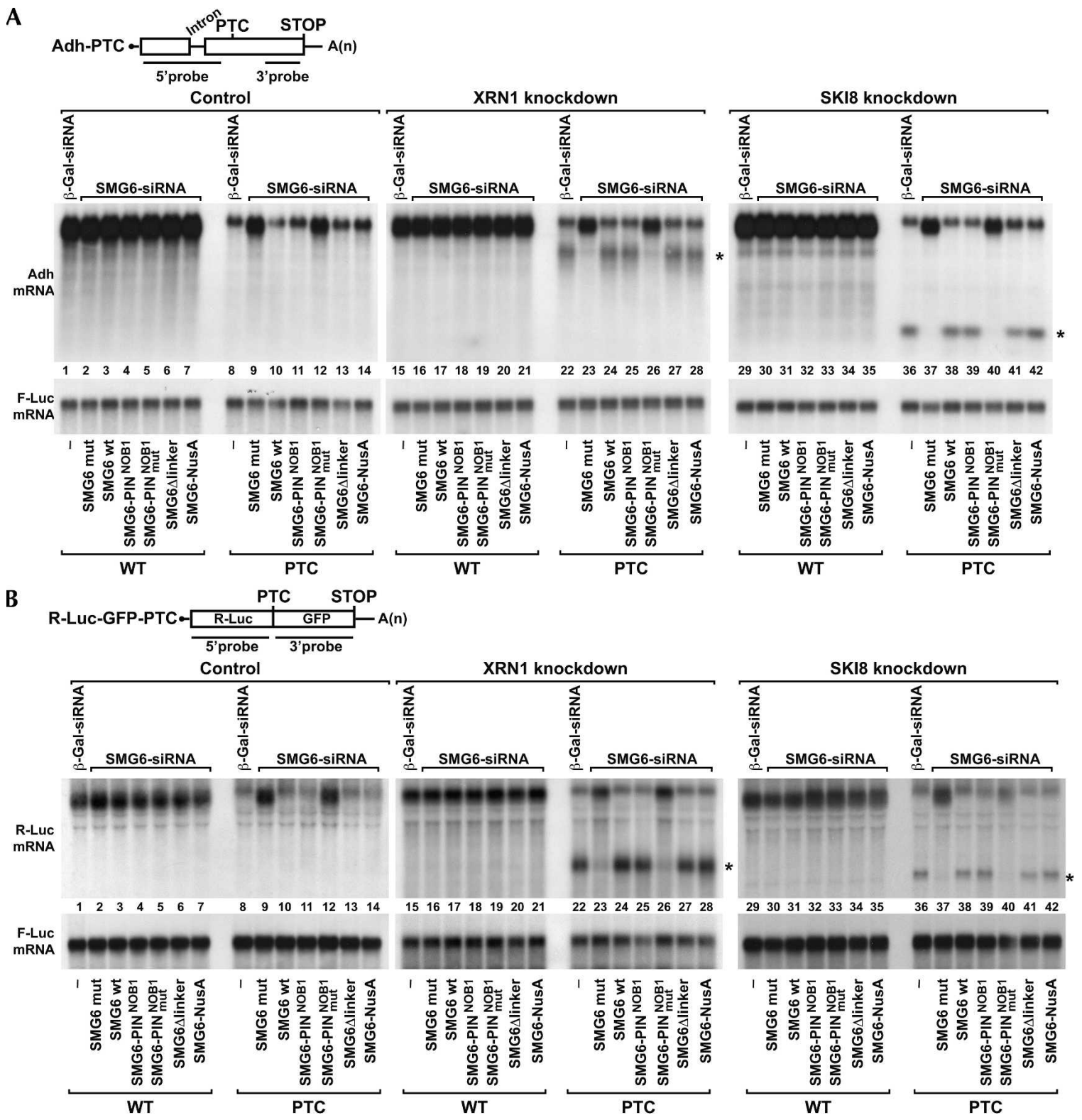

FIGURE 5. The accumulation of decay intermediates correlates with the catalytic activity of the SMG6-PIN domain. $(A, B)$ Control S2 cells or cells depleted of SKI8 or of XRN1 were transfected with the indicated siRNAs and the Adh $(A)$ or the R-Luc-GFP $(B)$ reporters. A plasmid encoding Firefly luciferase (F-Luc) served as a transfection control. Transfection mixtures also contained plasmids encoding siRNA-resistant versions of wild-type SMG6 or mutants as indicated. The asterisk shows the position of the Adh-PTC or R-Luc-GFP-PTC 5' and 3' decay intermediates. The position of the $5^{\prime}$ and $3^{\prime}$ probes used to detect the decay intermediates are indicated on the schematic representation of the Adh-PTC and R-Luc-GFP-PTC reporters, above the panels.

\section{A catalytically active SMG6-PIN domain is required for NMD in human cells}

The demonstration that SMG6 acts as an endonuclease raises the question of whether it fulfils the same role in other organisms for which degradation of nonsense mRNAs has been shown to occur by exonucleolytic digestion from both ends of the transcript (Lim and Maquat 1992; Chen and Shyu 2003; Lejeune et al. 2003; Conti and Izaurralde 2005; Lejeune and Maquat 2005). In particular, the PIN domain of human SMG6 is known to exhibit nuclease activity in vitro (Glavan et al. 2006), and SMG6 is required for efficient NMD in human cells (Gatfield et al. 2003; Paillusson et al. 2005; Luke et al. 2007). This prompted us to investigate the role of the SMG6-PIN domain in human cells. To this end, we used two well-characterized NMD reporters: one based on the $\beta$-globin gene (Thermann et al. 1998) and a second reporter based on the TCR- $\beta$ minigene (Wang et al. 2002). Using these reporters, we observed that human 
SMG6 carrying alanine substitutions of one (residue D1251; SMG6 mut-1) or two (residues D1251 and D1392; SMG6 mut-2) of the three catalytic aspartates inhibited NMD in a dominant negative manner, leading to an upregulation of the PTC-containing reporters within the twoto threefold range (data not shown). We reported similar values for PTC-containing transcripts in Drosophila S2 cells overexpressing a catalytically inactive SMG6 mutant (Glavan et al. 2006).

We next tested the human SMG6 mutants in a complementation assay similar to that described in Figure 1. The levels of the PTC-containing $\beta$-globin and TCR- $\beta$ mRNAs were strongly reduced in HeLa cells (down to $\sim 12 \%$ and $4 \%$ of the wild-type levels, respectively; Fig. 6A,B) as reported before (Thermann et al. 1998; Wang et al. 2002). Depleting SMG6 resulted in increased levels of the PTC-containing $\beta$-globin and TCR- $\beta$ reporters (five- and 14 -fold increase, respectively; Fig. 6A,B). The expression levels of the corresponding wild-type mRNAs were not significantly changed (Fig. 6A,B). Expressing an siRNAresistant version of wild-type SMG6 in cells depleted of endogenous SMG6 fully restored NMD (Fig. 6A,B). In contrast, the SMG6 proteins carrying mutations in catalytic residues failed to rescue NMD in cells depleted of endogenous SMG6 (Fig. 6A,B; SMG6 mut-1, SMG6 mut-2), although their expression levels were comparable to those of the wild-type (Fig. 6C). We conclude that, as in Drosophila S2 cells, degradation of PTC-containing mRNAs in human cells requires an active SMG6-PIN domain.

\section{Concluding remarks}

Taken together, our results provide compelling evidence that SMG6 is the long sought NMD endonuclease in $D$. melanogaster. SMG6 also contributes to the degradation of NMD targets in human cells (Gatfield et al. 2003; Paillusson et al. 2005; Luke et al. 2007), raising the question of its precise role in the NMD pathway, particularly in relation to SMG5 and SMG7, which are thought to trigger mRNA degradation by recruiting the general mRNA decay machinery (Unterholzner and Izaurralde 2004). One possibility is that SMG6 and the SMG5-SMG7 complex represent alternative decay pathways for NMD substrates in mammals (Luke et al. 2007). Regardless of the precise contribution of SMG6 to the degradation of PTC-containing mRNAs, our results show that metazoans have evolved a specific endonuclease that rids cells of mRNAs that terminate translation prematurely.

\section{MATERIALS AND METHODS}

\section{DNA constructs and NMD assays in D. melanogaster S2 cells}

The R-Luc-GFP reporter was constructed by inserting the GFP sequences (amplified from pEGFPC1, Clontech) into the XhoI site
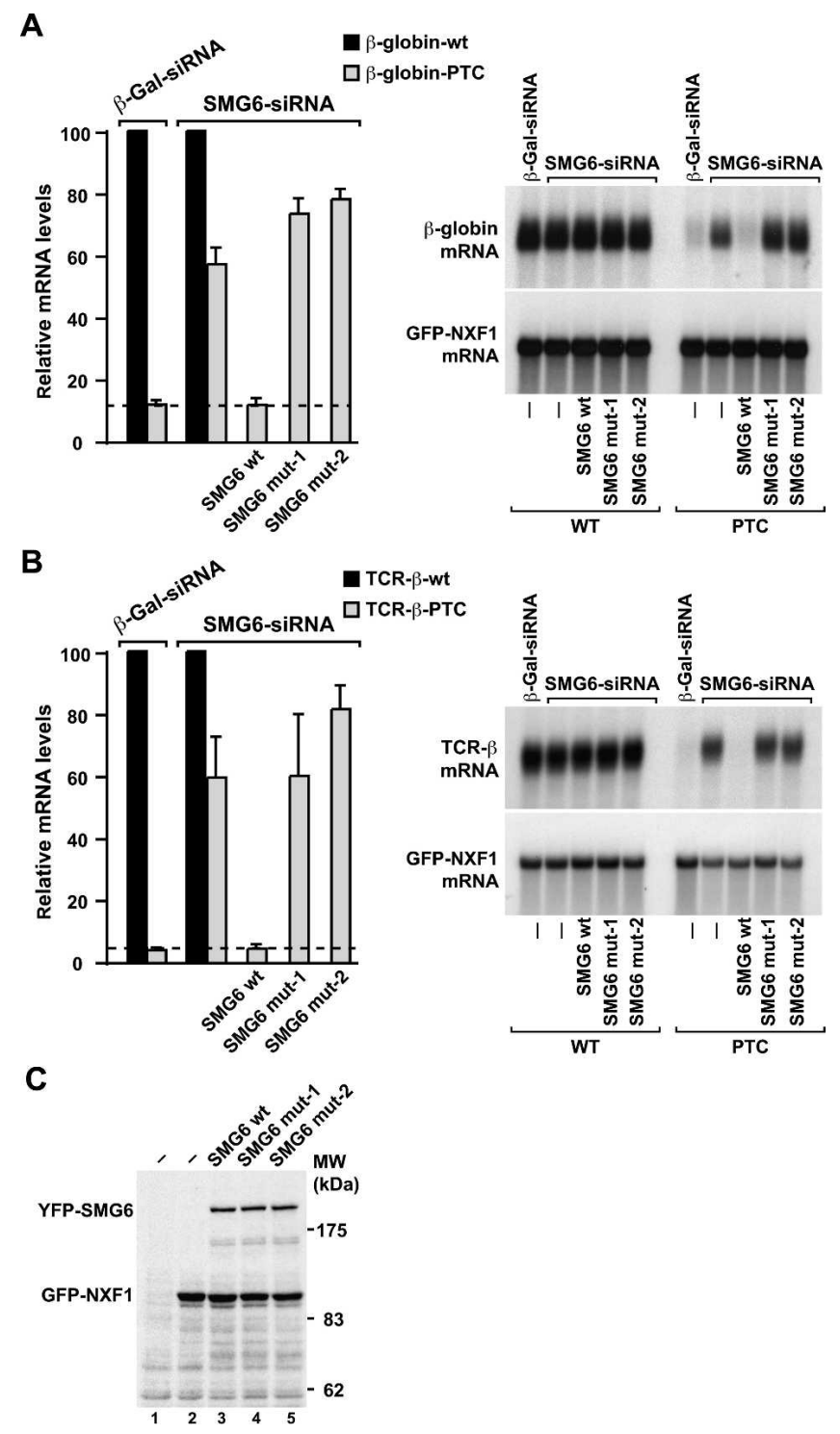

FIGURE 6. A catalytically active SMG6-PIN domain is required for human NMD. (A) HeLa cells were transfected with the indicated siRNAs plus a mixture of two plasmids: one expressing the $\beta$-globin reporter with or without PTC, and another expressing GFP-NXF1 as transfection control. Plasmids expressing siRNA-resistant versions of human SMG6 wild-type or mutants were included in the transfection mixtures, as indicated. These proteins are fused to yellow fluorescent protein (YFP). The levels of $\beta$-globin-wt or $\beta$-globin-PTC were analyzed by Northern blot and normalized to those of GFP-NXF1 mRNA. For each condition, the normalized values of the wild-type reporter were set to 100 (black bars). Mean values and SD from three independent experiments are shown. The right panels show Northern blot analysis of representative RNA samples. $(B)$ A similar experiment as described in $A$ was repeated using the TCR- $\beta$ reporter with or without PTC. RNA samples were analyzed as described in $A$. (C) The expression of YFP-SMG6 wild-type or mutants was analyzed by Western blotting using an anti-GFP antibody. Cotransfected pEGFPC1-NXF1 plasmid served as a transfection control. (Lane 1) Untransfected cells. (Lane 2) Cells transfected only with the pEGFPC1-NXF1 plasmid. 
of vector pAc5.1-R-Luc-V5, downstream from the R-Luc ORF. The 5' oligos used to amplify EGFP cDNA did, or did not, include an in-frame stop codon. The NMD reporters Adh-PTC and CATPTC were as described before (Gatfield et al. 2003; Gatfield and Izaurralde 2004; Behm-Ansmant et al. 2007). Plasmid pAc5.1Adh $\Delta$ (Behm-Ansmant et al. 2007) served as a transfection control.

D. melanogaster SMG5 and SMG6 cDNAs were cloned into a vector allowing the expression of $\lambda \mathrm{N}-\mathrm{HA}-$ peptide fusions (pAc5.1B- $\lambda$ N-HA; Glavan et al. 2006). The SMG6-PIN domain was deleted by mutagenesis using oligos that introduce a unique NotI site to generate a pAc5.1B- $\lambda$ N-HA-SMG6 $\Delta$ PIN plasmid. NOB1 cDNA (CG2972) or the SMG5-PIN domain were amplified by PCR from a $D$. melanogaster S2 cell cDNA library and cloned into the unique Not1 site of pAc5.1B- $\lambda$ N-HA-SMG6 $\Delta$ PIN construct in frame with the linker region. Protein mutants were generated by site-directed mutagenesis using the QuickChange mutagenesis kit from Stratagene, and the appropriate oligonucleotide sequences.

The SMG6 linker region (amino acids 676-728) was deleted by mutagenesis using oligos that introduced a unique Not1 site at this position to generate pAc5.1B- $\lambda \mathrm{N}-\mathrm{HA}-\mathrm{SMG} 6 \Delta$ linker. A fragment encompassing the NusA cDNA was amplified by PCR and cloned into the unique Not1 site of pAc5.1B- $\lambda$ N-HASMG6 $\Delta$ linker to generate pAc5.1B- $\lambda$ N-HA-SMG6-NusA. To substitute the SMG5-PIN domain with the PIN domain of SMG6, we first delete the SMG5-PIN domain by mutagenesis using oligos that introduced a unique Not1 site. The SMG6-PIN domain was amplified by PCR and cloned into this unique site in frame with the SMG5 linker region. All constructs were fully sequenced to confirm the presence of the mutations and the absence of additional mutations.

Transfections of S2 cells were performed in six-well plates using Effectene transfection reagent (Qiagen). The transfection mixtures contained $0.5 \mu \mathrm{g}$ of R-Luc-GFP reporter plasmids, $0.25 \mu \mathrm{g}$ of the F-Luc transfection control, and various amounts of plasmids expressing $\lambda \mathrm{N}-\mathrm{HA}$-protein fusions as indicated in the figures. When the Adh or CAT reporters were used, the transfection mixtures contained $0.2 \mu \mathrm{g}$ of wild-type or NMD reporters, $0.8 \mu \mathrm{g}$ of pAc5.1-Adh $\Delta$ plasmid, and $25 \mathrm{ng}$ of plasmids expressing $\lambda \mathrm{N}$ HA-protein fusions. The following siRNAs were used: $\beta$-Gal siRNA (5'-GCCAGUGAUACAGCGAA; Dharmacon) and SMG6siRNA (5'-UGAUUGAACAGGCCAUUAAUU). The siRNAs were transfected at a final concentration of $75 \mathrm{nM}$.

To deplete SKI8 or XRN1, D. melanogaster S2 cells were treated with a dsRNA targeting the first 700 nt of SKI8 or XRN1 ORFs, respectively. Thirty micrograms of dsRNA were used per six-well dish containing : $2 \times 10^{6}$ cells. Cells were treated with the dsRNA on days 0 and 4, transfected on day 6, and harvested on day 9 .

RNA samples were isolated $4 \mathrm{~d}$ after transfection using TriFast (Peqlab Biotechnologies) and analyzed as described before (Gatfield et al. 2003; Gatfield and Izaurralde 2004; Behm-Ansmant et al. 2007). Firefly and Renilla luciferase activities were measured $4 \mathrm{~d}$ after transfection using the Dual-Luciferase Reporter Assay System (Promega).

\section{DNA constructs and NMD assays in HeLa cells}

Human NMD reporters and the pEYFP-C1-SMG6 expression plasmid have been described before (Thermann et al. 1998; Wang et al. 2002; Gatfield et al. 2003; Unterholzner and Izaurralde 2004). The pEGFPC1-NXF1 (Gatfield et al. 2003; Unterholzner and Izaurralde 2004) plasmid served as a transfection control. SMG6 catalytical residues (D1251 and D1392) were substituted with alanines by site-directed mutagenesis using the QuickChange mutagenesis kit from Stratagene.

Transfections of human HeLa cells were performed in six-well plates, using Lipofectamine 2000 transfection reagent. The siRNAs were transfected at a final concentration of $70 \mathrm{nM}$ together with a mixture of three plasmids: one expressing the reporters $(0.5 \mu \mathrm{g}$ of $\beta$-globin with or without PTC, or TCR- $\beta$ with or without PTC), one expressing the transfection control $(0.25 \mu \mathrm{g}$, pEGFPC1NXF1), and a third plasmid ( $1 \mu \mathrm{g})$ expressing recombinant proteins or the corresponding empty vector. The following target sequences were used: SMG6 5'-AAGCCAGTGATACAGCGAATT, and the control $\beta$-Gal siRNA (see above). Cells were harvested $3 \mathrm{~d}$ after transfection. Total RNA was isolated using TriFast (peqlab biotechnologies) and analyzed as described before (Gatfield et al. 2003).

\section{Western blotting}

For Western blots total cell extracts were prepared by addition of protein sample buffer to cells $3 \mathrm{~d}$ (S2 cells) or $48 \mathrm{~h}$ (HeLa cells) after transfection. Proteins were separated by SDS-polyacrylamide gel electrophoresis and transferred to nitrocellulose membranes. Western blotting was performed with a rabbit polyclonal anti-HA antibody (1:1000; Invitrogen), anti-V5 antibodies (1:5000 Invitrogen), and rabbit polyclonal anti-GFP antibodies (1:5000) using the CDP-Star chemiluminescent immunoblot system (Western-Star kit from Tropix), as recommended by the manufacturer.

\section{ACKNOWLEDGMENTS}

We thank Andreas Kulozik, Matthias Hentze, and Miles F. Wilkinson for their generous gift of the $\beta$-globin and TCR- $\beta$ reporters. We are grateful to Isabelle Behm-Ansmant for her contributions during the initial phase of this project. This study was supported by the Max Planck Society and by a grant from the Deutsche Forschungsgemeinschaft (DFG, FOR855).

Received September 12, 2008; accepted September 26, 2008.

\section{REFERENCES}

Anders, K.R., Grimson, A., and Anderson, P. 2003. SMG-5, required for C. elegans nonsense-mediated mRNA decay, associates with SMG-2 and protein phosphatase 2A. EMBO J. 22: 641-650.

Behm-Ansmant, I. and Izaurralde, E. 2006. Quality control of gene expression, a stepwise assembly pathway for the surveillance complex that triggers nonsense-mediated mRNA decay. Genes \& Dev. 20: 391-398.

Behm-Ansmant, I., Gatfield, D., Rehwinkel, J., Hilgers, V., and Izaurralde, E. 2007. A conserved role for cytoplasmic poly(A)binding protein 1 (PABPC1) in nonsense-mediated mRNA decay. EMBO J. 26: 1591-1601.

Cali, B.M., Kuchma, S.L., Latham, J., and Anderson, P. 1999. smg-7 is required for mRNA surveillance in Caenorhabditis elegans. Genetics 151: 605-616.

Chen, C.Y. and Shyu, A.B. 2003. Rapid deadenylation triggered by a nonsense codon precedes decay of the RNA body in a mammalian 
cytoplasmic nonsense-mediated decay pathway. Mol. Cell. Biol. 23: 4805-4813.

Chiu, S.Y., Serin, G., Ohara, O., and Maquat, L.E. 2003. Characterization of human Smg5/7a, a protein with similarities to Caenorhabditis elegans SMG5 and SMG7 that functions in the dephosphorylation of Upf1. RNA 9: 77-87.

Conti, E. and Izaurralde, E. 2005. Nonsense-mediated mRNA decay: Molecular insights and mechanistic variations across species. Curr. Opin. Cell Biol. 17: 316-325.

Czaplinski, K., Ruiz-Echevarria, M.J., Paushkin, S.V., Han, X., Weng, Y., Perlick, H.A., Dietz, H.C., Ter-Avanesyan, M.D., and Peltz, S.W. 1998. The surveillance complex interacts with the translation release factors to enhance termination and degrade aberrant mRNAs. Genes \& Dev. 12: 1665-1677.

Denning, G., Jamieson, L., Maquat, L.E., Thompson, E.A., and Fields, A.P. 2001. Cloning of a novel phosphatidylinositol kinase-related kinase: Characterization of the human SMG-1 RNA surveillance protein. J. Biol. Chem. 276: 22709-22714.

Fatica, A., Oeffinger, M., Dlakić, M., and Tollervey, D. 2003. Nob1p is required for cleavage of the $3^{\prime}$ end of 18 S rRNA. Mol. Cell. Biol. 23: 1798-1807.

Fatica, A., Tollervey, D., and Dlakić, M. 2004. PIN domain of Noblp is required for D-site cleavage in 20S pre-rRNA. RNA 10: 1698-1701.

Fukuhara, N., Ebert, J., Unterholzner, L., Lindner, D., Izaurralde, E., and Conti, E. 2005. SMG7 is a 14-3-3-like adaptor in the nonsense-mediated mRNA decay pathway. Mol. Cell 18: 537-547.

Gatfield, D. and Izaurralde, E. 2004. Nonsense-mediated messenger RNA decay is initiated by endonucleolytic cleavage in Drosophila. Nature 429: 575-578.

Gatfield, D., Unterholzner, L., Ciccarelli, F.D., Bork, P., and Izaurralde, E. 2003. Nonsense-mediated mRNA decay in Drosophila: At the intersection of the yeast and mammalian pathways. EMBO J. 22: 3960-3970.

Glavan, F., Behm-Ansmant, I., Izaurralde, E., and Conti, E. 2006. Structures of the PIN domains of SMG6 and SMG5 reveal a nuclease within the mRNA surveillance complex. EMBO J. 25: 5117-5125.

Hodgkin, J., Papp, A., Pulak, R., Ambros, V., and Anderson, P. 1989. A new kind of informational suppression in the nematode Caenorhabditis elegans. Genetics 123: 301-313.

Houseley, J., LaCava, J., and Tollervey, D. 2006. RNA-quality control by the exosome. Nat. Rev. Mol. Cell Biol. 7: 529-539.

Kashima, I., Yamashita, A., Izumi, N., Kataoka, N., Morishita, R., Hoshino, S., Ohno, M., Dreyfuss, G., and Ohno, S. 2006. Binding of a novel SMG-1-Upf1-eRF1-eRF3 complex (SURF) to the exon junction complex triggers Upf1 phosphorylation and nonsensemediated mRNA decay. Genes \& Dev. 20: 355-367.

Lejeune, F. and Maquat, L.E. 2005. Mechanistic Links between nonsense-mediated mRNA decay and pre-mRNA splicing in mammalian cells. Curr. Opin. Cell Biol. 17: 309-315.
Lejeune, F., Li, X., and Maquat, L.E. 2003. Nonsense-mediated mRNA decay in mammalian cells involves decapping, deadenylating, and exonucleolytic activities. Mol. Cell 12: 675-687.

Lim, S.K. and Maquat, L.E. 1992. Human $\beta$-globin mRNAs that harbor a nonsense codon are degraded in murine erythroid tissues to intermediates lacking regions of exon I or exons I and II that have a cap-like structure at the $5^{\prime}$ termini. EMBO J. 11: 32713278.

Luke, B., Azzalin, C.M., Hug, N., Deplazes, A., Peter, M., and Lingner, J. 2007. Saccharomyces cerevisiae Ebslp is a putative ortholog of human Smg7 and promotes nonsense-mediated mRNA decay. Nucleic Acids Res. 35: 7688-7697.

Ohnishi, T., Yamashita, A., Kashima, I., Schell, T., Anders, K.R., Grimson, A., Hachiya, T., Hentze, M.W., Anderson, P., and Ohno, S. 2003. Phosphorylation of hUPF1 induces formation of mRNA surveillance complexes containing hSMG-5 and hSMG-7. Mol. Cell 12: 1187-1200.

Page, M.F., Carr, B., Anders, K.R., Grimson, A., and Anderson, P. 1999. SMG-2 is a phosphorylated protein required for mRNA surveillance in Caenorhabditis elegans and related to Upf1p of yeast. Mol. Cell. Biol. 19: 5943-5951.

Paillusson, A., Hirschi, N., Vallan, C., Azzalin, C.M., and Mühlemann, O. 2005. A GFP-based reporter system to monitor nonsense-mediated mRNA decay. Nucleic Acids Res. 33: e54. doi: 10.1093/nar/gni052.

Pal, M., Ishigaki, Y., Nagy, E., and Maquat, L.E. 2001. Evidence that phosphorylation of human Upf1 protein varies with intracellular location and is mediated by a wortmannin-sensitive and rapamycinsensitive PI 3-kinase-related kinase signaling pathway. RNA 7: 515.

Rehwinkel, J., Raes, J., and Izaurralde, E. 2006. Nonsense-mediated mRNA decay: Target genes and functional diversification of effectors. Trends Biochem. Sci. 31: 639-646.

Thermann, R., Neu-Yilik, G., Deters, A., Frede, U., Wehr, K., Hagemeier, C., Hentze, M.W., and Kulozik, A.E. 1998. Binary specification of nonsense codons by splicing and cytoplasmic translation. EMBO J. 17: 3484-3494.

Unterholzner, L. and Izaurralde, E. 2004. SMG7 acts as a molecular link between mRNA surveillance and mRNA decay. Mol. Cell 16: 587-596.

Wang, J., Vock, V.M., Li, S., Olivas, O.R., and Wilkinson, M.F. 2002. A quality control pathway that down-regulates aberrant T-cell receptor (TCR) transcripts by a mechanism requiring UPF2 and translation. J. Biol. Chem. 277: 18489-18493.

Yamashita, A., Ohnishi, T., Kashima, I., Taya, Y., and Ohno, S. 2001. Human SMG-1, a novel phosphatidylinositol 3-kinaserelated protein kinase, associates with components of the mRNA surveillance complex and is involved in the regulation of nonsense-mediated mRNA decay. Genes \& Dev. 15: 22152228 . 

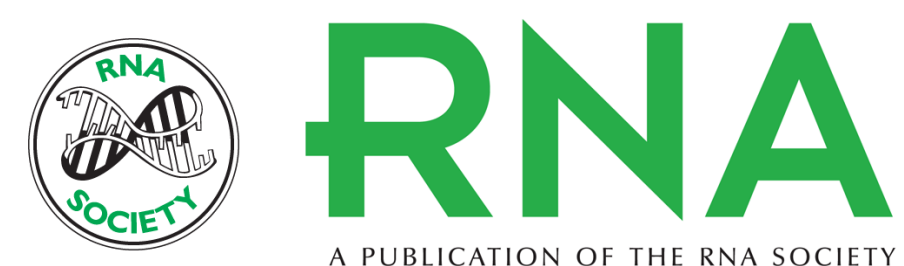

A PUBLICATION OF THE RNA SOCIETY

\section{SMG6 is the catalytic endonuclease that cleaves mRNAs containing nonsense codons in metazoan}

Eric Huntzinger, Isao Kashima, Maria Fauser, et al.

RNA 2008 14: 2609-2617 originally published online October 30, 2008

Access the most recent version at doi:10.1261/rna.1386208

$\begin{aligned} \text { References } & \begin{array}{l}\text { This article cites } 31 \text { articles, } 17 \text { of which can be accessed free at: } \\ \text { http://rnajournal.cshlp.org/content/14/12/2609.full.html\#ref-list-1 }\end{array} \\ \text { Open Access } & \text { Freely available online through the RNA Open Access option. } \\ \text { License } & \text { Freely available online through the open access option. } \\ \begin{array}{r}\text { Email Alerting } \\ \text { Service }\end{array} & \begin{array}{l}\text { Receive free email alerts when new articles cite this article - sign up in the box at the } \\ \text { top right corner of the article or click here. }\end{array}\end{aligned}$

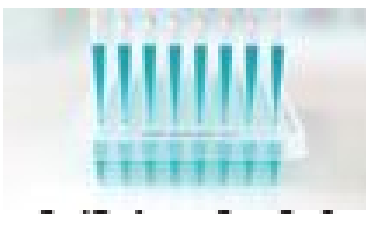

Providing Precise Solutions for your research.

To subscribe to RNA go to:

http://rnajournal.cshlp.org/subscriptions 